\title{
A TRANSFORMATION TO LINEARITY OF SOME NON-LINEAR DIFFERENTIAL-DIFFERENCE EQUATIONS
}

\author{
A. J. BRACKEN
}

(Received 12 June 1981)

(Revised 21 August 1981)

\begin{abstract}
The problem of solving a differential-difference equation with quadratic non-linearities of a certain type is reduced to the problem of solving an associated linear differentialdifference equation.
\end{abstract}

Consider differential-difference equations of the general form

$$
\dot{x}(t)=r(t) x(t)+x(t)[\theta(t) x(t)-\theta(t-1) x(t-1)],
$$

with $r$ and $\theta$ known functions, and $x$ an unknown function of the variable $t$. The class of equations so defined is rather restrictive, even though $r$ and $\theta$ are essentially arbitrary, but it is not without possible applications.

For example $x(t)$ could represent, in a continuous approximation, the size at time $t$ of a population whose growth is restricted by pairwise competition between its members, when members above a certain age do not compete amongst themselves. Were it not for this last condition, the familiar logistic equation

$$
\dot{x}(t)=r x(t)+\theta x(t)^{2},
$$

with $r$ (positive) and $\theta$ (negative) constants could be expected to define a useful deterministic model of the variation in time of the population size. The second term on the right-hand side of (2) models the effects of pairwise competition amongst all members. However, if members above a certain age do not compete amongst themselves, and if death of members has no significant effect on the population size during the period of interest, then a more appropriate competition term is

$$
\theta x(t)[x(t)-x(t-1)]
$$

(C) Copyright Australian Mathematical Society 1982 
with $\theta$ a negative constant. Here the term in square brackets represents, for a suitable scaling of $t$, the size of the subpopulation whose members are not above that certain age. Then in place of (2) one obtains equation (1), with $r$ and $\theta$ constant.

Equations of the form (1) can be linearized as follows. First, set

$$
y(t)=x(t) e^{-R(t)} \quad \text { where } R(t)=\int r(t) d t
$$

so that (1) is replaced by

$$
\dot{y}(t)=y(t)\left[\theta(t) e^{R(t)} y(t)-\theta(t-1) e^{R(t-1)} y(t-1)\right] .
$$

Next, write

$$
y(t)=f(t-1) / f(t) .
$$

(This may be compared with the substitution $y=\dot{f} / f$ which can be used to linearize a Riccati differential equation.) Then

$$
\dot{y}(t)=[f(t) \dot{f}(t-1)-\dot{f}(t) f(t-1)] / f(t)^{2},
$$

and (5) becomes

$$
\begin{aligned}
f(t) \dot{f}(t-1)- & \dot{f}(t) f(t-1) \\
& =\left[\theta(t) e^{R(t)} f(t-1)^{2}-\theta(t-1) e^{R(t-1)} f(t) f(t-2)\right],
\end{aligned}
$$

that is,

$$
\begin{aligned}
f(t)\left[\dot{f}(t-1)+\theta(t-1) e^{R(t-1)} f(t-2)\right] \\
=f(t-1)\left[\dot{f}(t)+\theta(t) e^{R(t)} f(t-1)\right]
\end{aligned}
$$

This has the general form

$$
f(t) g(t-1)=f(t-1) g(t)
$$

which implies

$$
g(t)=p(t) f(t)
$$

where

$$
p(t-1)=p(t)
$$

Thus

$$
\dot{f}(t)+\theta(t) e^{R(t)} f(t-1)=p(t) f(t)
$$

or equivalently

$$
\frac{d}{d t}\left[e^{-P(t)} f(t)\right]+\theta(t) e^{R(t)-P(t)} f(t-1)=0
$$


where

$$
P(t)=\int p(t) d t
$$

Since (12) holds,

$$
e^{P(t-1)-P(t)}=\lambda,
$$

with $\lambda$ constant, and so (14) can be written as

$$
\dot{h}(t)+\lambda \theta(t) e^{R(t)} h(t-1)=0
$$

where

$$
h(t)=e^{-P(t)} f(t)
$$

Because of (16),

$$
f(t-1) / f(t)=\lambda h(t-1) / h(t)
$$

and one can summarize by saying that solutions of (1) have the form

$$
x(t)=\lambda e^{R(t)} h(t-1) / h(t),
$$

where $\lambda$ is constant and $h$ satisfies (17).

Consider an initial value problem for (1). Suppose that $r(t)$ and $\theta(t-1)$ are continuous for $t \geqslant 1$, and that a function $x(t)$ is sought which is continuous for $0 \leqslant t<T$; which satisfies (1) for $1 \leqslant t<T$; and which, for $0 \leqslant t \leqslant 1$, equals a prescribed, continuous and everywhere non-zero function $\phi(t)$. More general conditions (on $\phi$ in particular) could be considered, but these will suffice for illustrative purposes. The value $\dot{x}(1)$ defined by (1) at $t=1$ must be interpreted as $\dot{x}(1+)$.

Then a function $h(t)$ should be sought, which is continuous for $-1 \leqslant t<T$; which is non-zero for $0 \leqslant t<T$; and which satisfies (17) for $0 \leqslant t<T$ and for a non-zero value of $\lambda$ to be determined. The initial data for $h$, that is, the value $\psi(t+1)$ of $h(t)$ for $-1 \leqslant t \leqslant 0$, must be determined by the initial data for $x$, as must the appropriate value of $\lambda$. Again, the value $\dot{h}(0)$ defined by (17) at $t=0$ will have to be interpreted as $\dot{h}(0+)$. The condition that $h$ should be non-zero for $0 \leqslant t<T$ may be regarded as determining the largest possible value of $T$ for which the problem under consideration is well-posed: at the smallest positive value of $t$ for which $h$ vanishes (if indeed $r, \theta$ and $\phi$ are such that a finite such value exists), then continuity of $x$ will fail by virtue of (20).

Now (20) implies that

$$
\phi(t)=\lambda e^{R(t)} \psi(t) / h(t) \text { for } 0 \leqslant t \leqslant 1 .
$$

From (17) and continuity of $h$ at $t=0$ one has

$$
h(t)=\psi(1)-\lambda \int_{0}^{t} \theta(\tau) e^{R(\tau)} \psi(\tau) d \tau \quad \text { for } 0 \leqslant t \leqslant 1,
$$


which, combined with (21), gives

$$
\phi(t)\left[\psi(1)-\lambda \int_{0}^{t} \theta(\tau) e^{R(\tau)} \psi(\tau) d \tau\right]=\lambda e^{R(t)} \psi(t) \quad \text { for } 0 \leqslant t \leqslant 1
$$

This is easily inverted to give

$$
\psi(t)=c \phi(t) \exp \left[-R(t)-\int_{0}^{t} \theta(\tau) \phi(\tau) d \tau\right] \text { for } 0 \leqslant t \leqslant 1
$$

and

$$
\lambda=\phi(1) \exp \left[-R(1)-\int_{0}^{1} \theta(\tau) \phi(\tau) d \tau\right] .
$$

From (21) and (22) one can see that the (non-zero) value of the arbitrary constant $c$ in (24) is immaterial: one can set $c=1$. Furthermore, the indefinite integral in (4) can of course be chosen such that $R(1)=0$ in (25), if desired. In this way the initial value problem for (1) is reduced to an initial value problem for (17), for a particular value of $\lambda$.

The solution of (17) can be investigated by known techniques. (See for example Bellman and Cooke [1].) In particular, when $r$ and $\theta$ are constants, so that $R(t)=r t$, the solution of (17) has been considered in detail by de Bruijn [2]. (See also Mahler [4] and Kato and McLeod [3].)

\section{Acknowledgement}

I am indebted to Dr. P. M. Diamond for a stimulating discussion.

\section{References}

[1] R. Bellman and K. L. Cooke, Differential-difference equations (Academic Press, New York, 1963).

[2] N. G. de Bruijn, "The difference-differential equation $F^{\prime}(x)=e^{\alpha x+\beta} F(x-1)$. I, II", Nederl. Akad. Wetensch. Proc. Ser. A 56 (also published as: Indagationes Math. 15) (1953), 449-458, 459-464.

[3] T. Kato and J. B. McLeod, "The functional-differential equation $y^{\prime}(x)=a y(\lambda x)+b y(x)$ ", Bull. Amer. Math. Soc. 77 (1971), 891-937.

[4] K. Mahler, “On a special functional equation”, J. London Math. Soc. 15 (1940), 115-123.

Department of Mathematics University of Queensland

St. Lucia

Queensland 4067 